\title{
TRATAMIENTOS NO CONVENCIONALES UTILIZADOS EN LA CURACIÓN DE LAS HERIDAS
}

\author{
Maira Alexandra Lozada Méndez, Nelson Rolando Campos Guzmán², Lorena Martínez \\ Delgado ${ }^{3}$
}

\section{Resumen}

El tratamiento de heridas crónicas es un desafío para los profesionales de la salud, por el tiempo de cicatrización, la lentitud del proceso, las recidivas y las repercusiones psicosociales: cambios en el estilo de vida, alteración en la convivencia familiar y autoimagen. En la actualidad existen diversos tratamientos para el manejo de heridas. Esta investigación tuvo como objetivo identificar tratamientos no convencionales utilizados en la curación de las heridas. Metodología: Revisión integrativa de literatura, que realizó una búsqueda en diferentes bases de datos: Science direct; Scopus, EBSCOhost; LILACS y PubMed. Se incluyeron artículos, publicados del 2001 al 2020, producto de investigación, ensayos clínicos y revisiones sistemáticas con Meta-análisis, en tres idiomas. Resultados: 51 artículos seleccionados utilizaron: manejo con miel, larvas, plantas medicinales y barro, entre otros. Conclusiones: Esta revisión permitió identificar diversos tratamientos y evidenciar el aumento en el número de investigaciones que buscan sustentación científica para la utilización de tratamientos no convencionales.

Palabras clave: cicatrización de heridas, terapias complementarias, técnicas de cierre de heridas.

Historial del artículo:

Fecha de recibido: 16-12-2020 - Fecha de aceptado: 25-02-2021

(C) 2021 Fundación Universitaria Juan N. Corpas. FUJNC.

Artículo Open Access bajo la Licencia Creative Commons Atribución-NoComercial-Compartirlgual 4.O Internacional.

DOI: 10.26752/revistaparadigmassh.v2i2.520

${ }^{1}$ Enfermera, UCI Adulto e intermedio, CAFAM, Especialista en Cuidado Crítico. Autor para correspondencia. Correo electrónico: maira_1795@hotmail.com

Enfermero, Especialista Enfermería Cardio Respiratorio, Magister en Epidemiología, Docente Programa Enfermería Universidad de Ciencias Aplicadas y Ambientales U.D.C.A. Correo electrónico: ncampos@udca.edu.co

${ }^{3}$ Enfermera, PHD Educación, Especialista en Docencia Universitaria, Diplomada en Educación para la Salud, Docente del Programa de Enfermería U.D.C.A. Correo electrónico: Imartinez@udca.edu.co 


\title{
NON-CONVENTIONAL TREATMENTS USED IN THE HEALING OF WOUNDS
}

\begin{abstract}
The treatment of chronic wounds is a challenge for health professionals, due to the time of healing, the slowness of the process, recurrences and the psychosocial repercussions: changes in lifestyle, alteration in family life and self-image. Currently there are various treatments for wound management. This research aimed to identify unconventional treatments used in wound healing. Methodology: Integrative literature review, which performed a search in different databases: Science direct; Scopus, EBSCOhost; LILACS and PubMed. Articles, published from 2001 to 2020, research product, clinical trials and systematic reviews with Meta-analysis, in three languages were included. Results: 51 selected articles used: handling with honey, larvae, medicinal plants and mud, among others. Conclusions: This review made it possible to identify various treatments and show the increase in the number of investigations that seek scientific support for the use of non-conventional treatments.
\end{abstract}

Keywords: wound healing, complementary therapies, wound closure techniques. 


\section{Introducción}

Este artículo, pretende mostrar los diferentes tratamientos no convencionales que han sido utilizados en la curación de las heridas. En la primera parte del artículo se presentan algunas de las ventajas de los tratamientos no convencionales y en los resultados se consolidan los artículos encontrados en la revisión en cuatro temáticas: el uso de la miel en las heridas crónicas y sépticas, la terapia larval en heridas crónicas, sépticas, y quirúrgicas; el uso de plantas medicinales en la curación de heridas crónicas, sépticas, y quirúrgicas y otros tipos de tratamientos utilizados en la curación de las heridas crónicas y sépticas como: camarones, terapia solar, y terapia con barro.

La curación de heridas es un tema tan antiguo como la historia del hombre. El hombre Neandertal en Irak 60.000 años A.C. usó hierbas para el manejo de los pacientes con quemaduras A.C; en el antiguo Egipto ya se usaban como apósitos el barro, gomas, resinas, miel, mirra y sustancias oleosas, la historia también relata que Hipócrates trataba las heridas con vino, cera de abejas, roble sagrado, aceite y azúcar, escuela que incluso se mantiene hasta nuestros días. (Andrades, P., Sepúlveda, S., \& Gonzáles, J. 2004).

En el artículo terapia tópica en el tratamiento de heridas crónica se define la herida como la ruptura de la integridad cutánea mucosa, que puede tener causas intencionales, traumáticas o isquémicas. La herida es considerada una pérdida de continuidad, que puede afectar desde la epidermis hasta estructuras más profundas de la piel. (Nicácio Da Silva, P., Alves de Almeida, O., \& Rocha, I. C. 2014).
Cuando se produce una herida, se desencadena un conjunto de procesos fisiológicos que utiliza el organismo para recuperar su integridad y arquitectura, que se conocen como proceso de cicatrización. El proceso de cicatrización es un fenómeno local del cual participan factores ambientales y fisiológicos que intervienen en el proceso de cicatrización y ejercen influencia en la calidad de la cicatriz. (Jimenez, C. E. 2008).

En este mismo sentido (Cook M, P. 2020), afirma que la cicatrización de heridas es un proceso complejo, compuesto de cuatro etapas, que puedeverse afectado por una serie de factores tanto ambientales como orgánicos y que, de acuerdo a la mayoría de los artículos analizados, la miel es un producto natural que da buenos resultados en el tratamiento de heridas y úlceras de diversa etiología, gracias a la multiplicidad de sus componentes.

En países en vía de desarrollo como Ghana, Mali, Nigeria y Zambia; o países latinoamericanos como Costa Rica, Nicaragua, República Dominicana están trabajando en una legislación que permita el uso de los tratamientos naturales, de allí radica la importancia de continuar con la investigación de las propiedades que tienen los tratamientos naturales para la curación de heridas.

Una revisión realizada por Israili ZH (2014) sobre las propiedades antimicrobianas de la miel, observó que los estudios clínicos han demostrado que la aplicación de miel a heridas cutáneas gravemente infectadas elimina rápidamente la infección de la herida y mejora la cicatrización del tejido. Un gran número de estudios clínicos limitados e in vitro han confirmado las propiedades antimicrobianas (antibacterianas, antifúngicas, antivirales 
y antimicobacterianas) de amplio espectro de la miel, pueden atribuirse a la acidez $(\mathrm{pH}$ bajo), efecto osmótico, alta concentración de azúcar, presencia de factores bacteriostáticos y bactericidas (peróxido de hidrógeno, antioxidantes, lisozima, polifenoles, ácidos fenólicos, flavonoides, metilglioxal y péptidos de abeja) y aumento de la liberación de citocinas y de las propiedades inmunomoduladoras y antiinflamatorias de la miel; la acción antimicrobiana involucra varios mecanismos.

Esta revisión tuvo como objetivo identificar los tratamientos no convencionales más utilizados en la curación de las heridas y los aportes presentados en los artículos por los autores frente al uso de los mismos.

\section{Materiales y métodos}

Se realizó una revisión integrativa a partir de una pregunta de investigación ¿Cuáles son los tratamientos no convencionales, más utilizados en la curación de heridas en adultos del 2001 al 2020? Y una búsqueda de artículos científicos en las bases de datos: Science Direct; Scopus, EBSCOhost; Directory of Open Access Journals, LILACS y PubMed (Medline). Como términos DeCS se emplearon: cicatrización de heridas; terapias complementarias, técnica de cierre de heridas.

Los artículos revisados incluyeron: artículos resultados de investigación, ensayos clínicos y revisiones sistemáticas con Meta-análisis, no se incluyeron estudios de caso. Los criterios de inclusión fueron artículos publicados del año 2001 al 2020; en idioma español, inglés y portugués; población objeto: adultos; artículos relacionados con heridas crónicas vasculares de tipo arterial y venoso, pie diabético, heridas quirúrgicas, sépticas, y tratamientos no farmacológicos a los tipos de heridas ya mencionados entre ellos (miel, larvas, plantas, peloidoterapia y terapia solar). Los artículos fueron codificados, y clasificados en una matriz de Excel, que contenía la siguiente información: base de datos, definición de las fuentes de búsqueda, DeCS (tres idiomas), ecuaciones booleanas empleadas, tratamiento empleado: ventajas, desventajas, usos recomendados y los criterios de inclusión ya mencionados.

\section{Resultados}

\section{Tratamiento de las heridas con miel}

La miel es el biomaterial más antiguo usado como apósito de las heridas y la efectividad de la miel en la gestión de heridas ha sido confirmada por muchos estudios. No hay reacción alérgica y no tiene efectos secundarios significativos debido a la aplicación clínica de la miel. Ésta tiene todos los criterios beneficiosos, pues la miel se podría utilizar como una opción alternativa adecuada en la mayoría de las heridas infectadas debido a sus propiedades anti-bacterianas y rentables. Así mismo, varios estudios han documentado que, en comparación con los apósitos convencionales, la miel ha mostrado una eficacia significativamente mayor para el tratamiento de heridas (Orellana, E., y Encalada R, S, 2019). Sin embargo, los autores Oryan, A., Alemzadeh, E., \& Moshiri, A. (2015), consideran que los médicos deberían ser conscientes sobre la composición y las propiedades biológicas de la miel seleccionado antes de la aplicación médica.

La miel, además de poseer una amplia actividad antimicrobiana contra diversas bacterias y hongos, presenta una excelente capacidad antioxidante, activando la línea monocítica 
y factores antiinflamatorios. (Schencke, C., Salvo, J., Vasconcellos, A., \& Sol, M. d. (2013), Bang L, Bunting C, Molan P. (2003), Tonks AJ, Cooper RA, Jones KP, Blair S, Parton J, Tonks A. (2003) y Buratti S, Benedetti S, Cosio. (2007)). Estudios con la miel evidencian histológicamente suactividad antiinflamatoria demostrando que puede ser tan efectiva como la prednisolona, en evidencia de lo anterior el artículo: Could Honey Have a Place in Colitis Therapy? Effects of Honey, Prednisolone, and Disulfiram on Inflammation, Nitric Oxide, and Free Radical Formation (Bilsel, Y., Bugra, D., Yamaner, S., Bulut, T., Cevikbas, U., \& Turkoglu, U. ,2002) concluyendo que la miel disminuye el estrés oxidativo generado por la liberación de radicales libres de las heridas y pronta cicatrización de la herida.

Se plantea que la miel es una sustancia que provee un ambiente húmedo a la herida, de esta manera se convierte en un medio idóneo para iniciar la recuperación del tejido, así las células epiteliales son capaces de crecer sobre la superficie de la herida y los fibroblastos pueden contraerse para aproximar los bordes de la lesión, al mismo tiempo las actividades antimicrobianas de la miel evitan la proliferación de microorganismos en el lecho húmedo de la herida. (Girao, R. A. 2014 y Campos, D. C., Graveto, J. M., \& Silva, M. A. ,2009)

Por otra parte, en la revisión se encuentra un ensayo clínico aleatorio llevado a cabo en el Clinical Nurse Specialist in Leg Ulcer Care Aintree University Hospitals NHS Foundation Trust, Liverpool, UK (Robson, V., Dodd, S., \& Thomas, S. 2009) que contrastó el uso de métodos de tratamiento convencionales, y el uso de miel en 114 pacientes con heridas crónicas, obtuvo como resultado que la tasa de reducción del 50\% de la herida crónica a las 12 semanas fue: 68.2\% P 0.266 IC 95\% (54.4\% -81.2\%) en el grupo tratado con miel y $70.5 \%$ P 0.266 IC 95\% (57,1\%-82.8\%) en el grupo convencional; y la tasa de reducción del 50\% de la herida crónica a las 24 semanas fue: 94.0\% (79.1\% a 99.4\%) a los tratados con miel, y en el grupo control (terapia convencional) fue $80.1 \%$ (66.4\% a 90.9\%). En conclusión, el tiempo medio de reducción del 50\% en el área de la herida se produjo a los 32 días en el grupo tratado con miel, en comparación con 46 días en el grupo de control (tratamiento convencional). (Robson, V., Dodd, S., \& Thomas, S. 2009)

En otro estudio cuantitativo (Calderon, $\mathrm{M}$. d., Figueroa, C. S., Arias, J. S., Sandoval, A. H., $\&$ Torre, F. O. 2015) combinaron la miel de Ulmo con ácido ascórbico para la curación de ulceras venosas crónicas en 18 pacientes de ambos sexos, con edad promedio de 64 años y 13 meses en promedio de antigüedad de la lesión; las curaciones, se realizaron con la aplicación tópica de miel de Ulmo y de manera paralela consumo oral diario de ácido ascórbico de 500 mg.; finalizado el estudio, se observó la curación total de las úlceras venosas, en el total de los pacientes participantes en el estudio. (Calderon, M. d., Figueroa, C. S., Arias, J. S., Sandoval, A. H., \& Torre, F. O. (2015)

Tradicionalmente se ha considerado que la miel tiene efectos antimicrobianos. Un estudio realizado por Abd Jalil MA, Kasmuri AR, Hadi H (2017), mostró que el antioxidante de la miel de abeja sin aguijón podría romper la cadena de radicales libres que provocan un efecto perjudicial en la zona herida. Además, las propiedades antimicrobianas de la miel de abeja sin aguijón podrían superar 
la contaminación bacteriana y mejorar así la tasa de curación. y así mismo puede ayudar con la eliminación de los agentes patógenos en una herida, por ello una herida se considera infectada cuando existe presencia de 100.000 colonias o más de microorganismos por gramo de tejido y signos claros de osteomielitis, infección regional o general, (Muñoz GF. 2006) Además es importante resaltar que, si una herida infectada no es tratada de manera adecuada, puede convertirse en herida crónica ya que la infección retrasa la cicatrización porque prolonga la fase catabólica e inhibe la proliferación de tejido conjuntivo, así como los restos necróticos y cuerpos extraños. (Gentil Gl, Smirova P 2009)

Por lo anterior, (Lavandera RI. 2011) se incluyó un ensayo clínico terapéutico que tuvo como objetivo determinar la acción de la miel en el tratamiento de heridas sépticas, en una población de 200 pacientes entre 20 y 40 años, 123 del sexo masculino y 77 del femenino. Después de haber realizado el estudio, se evidenció que: "en el grupo experimental en las primeras 24 horas de tratamiento, desaparece prácticamente la fetidez y el tejido desvitalizado, mientras que esta situación se prolongó en el grupo de control hasta 5 o 6 días después de iniciado de tratamiento". Además, anotan que "La miel atraemacrófagosque participanenla"limpieza de la herida", acelera el desprendimiento del tejido desvitalizado, necrótico o gangrenoso, provee una fuente de energía local y forma una capa proteica protectora en la herida.

En contraposición a lo anterior Bradshaw en el 2011 hizo una comparación de actividad antimicrobiana in vitro en una gama de apósitos antimicrobianos compuestos por miel, yodo y plata, en el análisis de los datos se obtuvo que no hubo diferencia significativa en el tamaño de la zona de inhibición (ZOI) utilizando el de caldo comparación con fluido de simulación de la herida (SWF) para la miel o plata respectivamente $P$ (0.981-0.567); sin embargo, una diferencia significativa fue observada para el yodo P(0.005). Es decir, se recomienda que el profesional de la salud, haga una evolución exhaustiva antes de realizar la asignación de un tratamiento, y de igual forma se ve que el yodo mostró mayor actividad antimicrobiana y por ende la curación de la herida fue mayor. (Bradshaw, C. E. 2011)

Apoyando el postulado anterior, Marshall C, Queen J, Manjooran J. (2005) realizaron un estudio pragmático que incluyo 51 pacientes asignados al azar para recibir tratamiento con yodo o miel. La media de la curación de los tiempos era de 33 días En total las heridas curadas por validez estadística curaron más rápido con yodo (30 días) en comparación con miel (44 días), pero no hubo ninguna diferencia significativa en el tiempo de curación para las heridas por avulsión parcial (18 días con miel vs 23 días con yodo); todavía que la diferencia no es estadísticamente significativa, además se ha mostrado que no hay diferencia estadísticamente significativa entre el uso de apósitos de hidrogel y el uso de la apifarmacoterapia en el tratamiento de abrasiones $\mathrm{P}$ (0.75) (16,48 días vs 16,88 días). (Ingle R, Levin J, Polinder K. 2006).

Aunque las soluciones yodadas han sido ampliamente usadas para la curación de las heridas, se han documentado algunos efectos nocivos del yodo sobre la piel, ya que por su composición química tiene resultados corrosivos en la piel que pueden llegar a causar quemaduras, alterar la irrigación sanguínea, no logrando el efecto terapéutico 
deseado. Por ello se recomienda que antes de realizar la asignación de un tratamiento con soluciones yodadas, se haya realizado una evaluación exhaustiva de otros tratamientos para la curación de las heridas con el fin de garantizar la óptima evolución de la herida.

La miel es una sustancia natural, rica en nutrientes que puede contribuir a la curación de la herida de diversas formas, sobre todo al actuar bloqueando la actividad de los microorganismos invasores y manteniendo un ambiente húmedo en la herida, de esta manera se puede mantener la herida libre de infecciones y la epitelización continua, logrando la integridad cutánea; también es importante resaltar que este tratamiento es útil en las heridas que no cierran por primera intención debido a los factores que la preceden (cronicidad e infección) actuando sobre éstos y mejorando la maduración en el proceso de cicatrización; por otra parte no sé evidenció diferencias significativas relacionadas con otros tratamientos durante la revisión (yodo, plata), sin embargo consigue ser una opción terapéutica efectiva que se puede recomendar al usuario del servicio de salud con el fin de mejorar la atención y la calidad de la prestación de los servicios, incluyendo las practicas ancestrales seguras en los tratamientos llevados a cabo para un estado de salud óptimo en los pacientes.

\section{Tratamiento con larvas}

Se afirma que el uso de las larvas promueve un rápido desbridamiento, facilitan la remoción de bacterias patógenas, reducen el mal olor, y en algunos casos el dolor (Ávila, C. M., \& Vázquez, R. T. 2006), además se realizó un estudio de cohorte controlado de 18 pacientes con diabetes (Sherman, R. A. (2013) y Richardson, M. (2004)), que manifestaban un total de 20 heridas en los pies y las piernas que no sanan en adultos masculinos; 6 heridas fueron tratadas con terapia convencional, 6 con terapia larval, y 8 con terapia convencional seguida de la terapia larval. Los resultados obtenidos muestran la terapia larval se asoció con más rápido desbridamiento y cicatrización de la herida que la terapia convencional. Las heridas tratadas con terapia larval tuvieron una reducción del tejido necrótico de un 50\% en 9 días, mientras que las heridas tratadas con terapia convencional no alcanzaron la reducción del tejido necrótico del 50\% hasta el día 29, P (0,001). Dentro de 4 semanas, las heridas fueron completamente desbridadas con la terapia larval, mientras que las heridas tratadas con la terapia convencional en un promedio de 5 semanas todavía estaban cubiertas con tejido necrótico en más del $33 \%$ de la superficie de la herida P $(0,001)$. En conclusión, la terapia larval puede acelerar la curación de las heridas, al promover la formación de tejido de granulación, la remoción del tejido necrótico y la curación de la herida. (Sherman, R. A. 2013).

Se ha observado que si bien la terapia larval es un método de desbridamiento simple, eficaz, selectivo, rápido y seguro para el tratamiento de heridas crónicas, debido a que actúa a nivel del desbridamiento, desinfección y cicatrización de la herida, la terapia larval está relacionada con un aumento del nivel del dolor, por lo que se recomienda pautar analgesia. Debido a la repulsión que generan las larvas en muchas personas, es muy importante informar a los pacientes sobre las ventajas y desventajas de esta terapia para evitar el rechazo, como lo menciona (Rodriguez, D., 2020) en su revisión. 
La terapia larval (TL) es la aplicación de larvas de la mosca Lucilia Sericata en forma estéril en una herida para su pronta cicatrización; por ello en una revisión de literatura realiza sobre el tratamiento de las heridas crónicas mediante larvas de moscas Corónida Verde: (Ávila, C. M., \& Vázquez, R. T. 2006) se encuentra que únicamente una minoría de las 80.000 especies de mosca poseen propiedades que permiten su uso médico, entre ellas, la larva de la mosca Corónida verde que se utiliza de forma habitual en la actualidad. El tratamiento de las heridas crónicas con larvas, ha demostrado que es una alternativa terapeútica que permite que las heridas se mantengan limpias y libres de tejido necrótico. En este apartado, se discutirán las propiedades que tiene el uso de larvas en la curación de heridas crónicas y sépticas. (Jarczyk, G., Jackowski, M., Szpila, K., Boszek, G., \& Kapelaty, S. 2008).

Un estudio realizado por el Departamento de Ciencias Farmacéuticas de la Universidad de Nottingham (Horobin Aj, Shakesheff Km, Woodrow S, Robinson C, Pritchard Di. 2003) tuvo como objetivo "investigar los efectos de los productos de excreción y secreción de la larva L. Sericata (ES) sobre el comportamiento de los fibroblastos, que se sembró sobre la superficie de los componentes de matriz extracelular (ECM) y colágeno. De esta investigación se obtuvo que "las secreciones de las larvas de L. Sericata modifican la adhesión de fibroblastos y la difusión a través de la superficie de las proteínas de la matriz extracelular, mientras que mantiene las células viables con cualquiera de las larvas $P(<0,01)$.

De igual forma el estudio realizado por Téllez, G. A., Acero, M. A., Pineda, L. A., \& Castaño, J. C. (2012) tuvo como objetivo documentar el efecto de la larvaterapia por medio de los índices PUSH y Wound Bed Score, allí quedó demostrado que con solo una aplicación de la larvaterapia hay una mejoría del aspecto de la herida y en los puntajes evaluados; los resultados arrojaron que el promedio de curación de la herida con el índice PUSH fue de (12.0 a 9.7 Puntos) donde hubo una disminución de 2,3 puntos, en promedio, y con el Wound Bed Score se obtuvo, un incremento de 2,7 (11.3-14) puntos, lo que demuestra una recuperación en ambas escalas, de este modo muestran que el tratamiento con larvas es un método de desbridamiento rápido comparado con los tratamientos no quirúrgicos, que acelera la curación total de la herida.

El estudio Maggot debridement therapy of infected ulcers: patient and wound factors influencing outcome a study on 101 patients with 117 wounds (Steenvoorde, P., Jacobi, C. e., Doorn, L. V., \& Oskam., J. 2007). En el resultado de las 116 heridas tratadas con larvas, 78 heridas $(67,2 \%)$ tuvieron resultados beneficiosos y 38 heridas (32,8\%) tuvieron resultados fallidos este estudio concluye, la terapia larval es una técnica que funciona a la hora de llevar a cabo un tratamiento para la curación de una herida, sin embargo, hay que revisar las característica de ésta, así se puede obtener la mejoría rápida y efectiva de la herida.

En un estudio realizado en el año 2000, cuyo objetivo fue comparar la terapia de desbridamiento con larvas (LDT) y apósitos de hidrogel en el tratamiento de úlceras venosas necróticas en 12 pacientes, los resultados mostraron que el costo del tiempo de enfermería fue significativamente mayor en el grupo estándar en comparación con el grupo LDT. (Costo promedio de £53.85 
vs $£ 10,70)$. En general la mediana del costo del tratamiento del grupo larval es £18.64 en comparación con £136.23 para el grupo control P $(<0,05)$. Este pequeño estudio demuestra claramente la mejora en el tiempo requerido para desbridar las úlceras usando LDT, que esta terapia es rentable, y que reducirían significamente los costos y el tiempo en la curación del tratamiento. (Waymant, J., Nirojogit, V., Walker, A., Sowinskia, A., \& Walkera, M. A. 2000).

Para la curación de las heridas crónicas las larvas tienen múltiples beneficios, como se ha descrito anteriormente poseen variados efectos a través de las cuales puede restaurar la integridad de la piel de manera progresiva, y de este modo evitar complicaciones como (amputaciones, mala cicatrización, entre otros.); lo que se destaca es la capacidad de fomentar la producción de tejido de granulación por la migración rápida y efectiva de los fibroblastos; y el desbridamiento rápido de tejido necrótico que impide la formación del nuevo tejido, estas propiedades se deben al uso de una serie de enzimas y factores propio de las larvas para destruir el material biológico que no es óptimo para la recuperación de la integridad cutánea.

A raíz de los resultados de los estudios de los últimos años, realizados en diversos países (China, Reino Unido, Irán, España, Brasil, Estados Unidos, Portugal) se puede dilucidar el efecto de la terapia larval sobre las heridas. Esta terapia es eficaz como desbridante, acelera la curación y tiene efecto antibacteriano. (Martínez, P. S., 2020). Además, representa una buena alternativa para minimizar los costos de los tratamientos, y el tiempo invertido por parte del personal de enfermería para curar una herida. Es importante que continúe la investigación en este tipo de alternativas terapéuticas, ya que son tratamientos naturales que pueden potenciar la curación de las heridas y de igual forma, es significativo realizar la divulgación de estos de estudios, resaltando la importancia de estos tratamientos con fines terapéuticos reduciendo estigmas sociales y potenciando la adherencia a los tratamientos.

\section{Tratamiento con plantas}

El uso de las plantas con fines medicinales se remonta al principio de la historia de la humanidad: 3.000 años antes de Cristo se escribió el libro más antiguo sobre plantas medicinales en China; los sumerios, 2.500 años a.C., usaron las plantas con fines curativos; los asirios conocieron un poco más de 250 hierbas medicinales. En nuestro país, esta práctica tiene sus raíces en una riquísima herencia cultural, gracias al legado de diversas culturas (indígenas, africanas y europeas) que han utilizado estas plantas con fines rituales, medicinales y gastronómicos. (Ministerio de Agricultura y Desarrollo Rural Colombia, 2011)

En primera instancia se documenta una revisión sistemática de la literatura cuyo objetivo fue proporcionar una visión general de las propiedades de la celulosa bacteriana o nata de coco (BC). La BC es un polímero producido por algunas bacterias y su uso como un material de curación de heridas se rige por sus peculiares características: tiene una resistencia a la tracción, flexibilidad y retención de agua, una permeabilidad pronunciada a gases y líquidos, y una gran compatibilidad con los tejidos vivos. En conclusión, los autores refieren que la BC es una opción económica, que comparte características similares con los demás apósitos que están a la vanguardia, 
convirtiéndose en una opción rentable, y eficaz para la curación de heridas crónicas, por su alta biocompatibilidad, y modificaciones favorables en el proceso de biosíntesis de nuevo tejido. (Sulaeva, I., Henniges, U., Rosenau, T., \& Potthast, A. 2015)

Así mismo se llevó a cabo un estudio descriptivo y prospectivo (Silva, R. S., Matos, L. S., Araújo, E. C., Paixão, G. P., Costa, L. E., \& Pereira., Á. 2014), en una Unidades Básica de Salud de la Familia (USF) en la ciudad Senhor do Bonfim - Bahía Noreste de Brasil, que tuvo como propósito conocer las prácticas populares frente a la curación de las heridas. En el estudio se pudo observar que los cuidados populares se realizaron con plantas medicinales. Después de llevada a cabo la investigación, evidenciaron que la granada, los extractos de anacardo y jurema, el uso la mastruz, la leche mangaba,jurubeba, cedro, los carobinha, lentisco y el drenaje de las patatas, serían las plantas que la comunidad utiliza comúnmente en el tratamiento de las heridas, han resultado beneficiosas, y han sido parte de sus prácticas de cuidado para la curación de las heridas (agudas o crónicas). La conclusión a la que llegaron fue que existe la necesidad de una mayor atención por las enfermeras en las prácticas de valoración popular, con el fin de respetar los aspectos culturales y generacionales, que se contemplan en la teoría de la transculturalidad, propuesta por Leininger. (Leininger, M. 1999)

Una investigación utilizó una fórmula de hierbas Astragali Radix Radix (la raíz de Astrágalo) y Rehmanniae (remaniha glutinosa) sobre la piel, de personas que tenían algún tipo de herida, el objetivo era identificar la eficacia de estas plantas con respeto a la actividad fibroblástica y la regeneración del tejido, los resultados mostraron que la migración de los fibroblastos juega un papel esencial en la reparación de heridas. (Zhang, Q., Fong, C. C., Yu, W. K., Chen, Y., Wei, F., Koon, C. M., ...Yang., M. 2012)

Por otra parte, Andrade, A. P. (2012) llevo a cabo una investigación enfocada a establecer las bases etnobotánicas para evaluar el potencial del marañón (Anacardium occidentale L.) como cicatrizante de heridas y analizar los datos botánicos y antropológicos para el desarrollo del proceso de validación como planta medicinal cicatrizante. La conclusión a la cual llego el estudio es que, tomando las hojas, el tallo, o la cascara del fruto, en cualquier forma de preparación se puede llegar a la cicatrización continua de la herida disminuyendo la extensión y los signos clínicos de infección.

Piriz, M., Lima, C., Jardim, V., Mesquita, M., Souza, A., \& Heck, R. (2014). En una revisión que tuvo como objetivo identificar las plantas utilizadas para la curación de heridas (crónicas, agudas) y quemaduras, los investigadores obtuvieron como resultado el estudio de un total de 52 plantas medicinales y un compuesto de hierbas de forma experimental o clínica, ya que sus efectos sobre la ayuda de proceso de curación, con la mayoría (88,5\%) mostraron una eficacia. Por lo tanto, se verifica que el uso de plantas medicinales es una alternativa importante para el tratamiento de heridas, que pasa a formar parte de atención en el sistema de salud de Brasil, lo que sugiere nuevos estudios pruebas clínicas, los costos y beneficios y la actualización constante de las publicaciones realizadas. Así mismo una investigación cuyo objetivo fue evaluar los potenciales para el uso de hojas de la planta Aspilia africana en 
el cuidado de heridas, el resultado fue que el extracto y las fracciones reducen el tiempo de sangrado de forma significativa $(p<0,05)$ y el tiempo de coagulación de la sangre, de esta forma los autores afirman que las hojas de A. Africana poseen componentes capaces de detener el sangrado de la herida, inhibir el crecimiento de contaminantes microbianos de heridas y acelerar la cicatrización. (Okoli, C., Akah, P., \& Okoli2, A. 2007).

Frente a la cicatrización, un estudio realizado por (Inocente, C. M. A., 2020) demostró que los extractos hidroalcohólicos de Annona muricata L. (guanábana) al $25 \%$, Opuntia ficusindica L. Mill. (tuna) al 25 \% y Musa acuminata Colla (plátano bellaco) al $4 \%$ presentan actividad cicatrizante con proyección similar al gel referencia de marca comercial. Enel caso de los extractos hidroalcohólicos de Urtica Urens L. (ortiga) al $10 \%$, Peperomia congona Sodiro (congona) al $25 \%$ y Ormosia Coccinea (Aubl) Jacks (huayruro) al $2 \%$, presentan actividad cicatrizante similar al proceso de cicatrización normal fisiológico, en heridas incisas de Rattus norvegicus albinus (rata albina). (Arenas, D. J. D., 2019), en su tesis logró evaluar el efecto cicatrizante del extracto etanólico de Annona muricata L. encontrando mejor efecto con una concentración al 100\%. En condiciones experimentales se ha demostrado que el extracto etanólico de las hojas de Annona muricata L. (Guanábana) al ser administrado por vía tópica posee efecto cicatrizante al ser aplicado sobre heridas superficiales inducidas en Mus musculus var albinus.

La terapia con plantas ha sido ampliamente utilizada desde tiempos remotos, por lo que se ha convertido en una estrategia para la población que permite acercarse sin mayor dificultad a los tratamientos para el cuidado de la salud. (Campos, D. C., Graveto, J. M., \& Silva, M. A. 2009) afirman que las plantas tienen efectos que permiten propiciar un ambiente favorable para la curación de las heridas, son útiles como emolientes, antibacteriano, antiséptico, la promoción del tejido epitelial y la remoción del tejido necrótico. Es importante que, a nivel nacional, se investigue sobre propiedades de las plantas nativas, a fin de garantizar el uso seguro de plantas con propiedades curativas por parte de las poblaciones.

\section{Otros tratamientos empleados en la curación de heridas}

Las terapias alternativas, son un método ampliamente utilizado hoy en día para todo tipo de afecciones al ser humano en este caso las heridas de tipo crónico. Se han identificado terapiasquepermitenlacuración delas heridas y que han sido de menor auge científico, pero que aun así muestran resultados favorables para sanar las heridas, a continuación, se describen estos tratamientos.

La investigación para la cicatrización de las heridas que fue el Ilevado a cabo por Sánchez, H. F., Lozano, A. Y., Méndez-Gómez, E., Vázquez, A. E., Hernández, M. d., \& Lizárraga, A. E. (2014) tuvo como objetivo evaluar in vivo el efecto cicatrizante de un gel de quitosano obtenido de exoesqueleto de camarón blanco Litopenaeus vannamei, que ayuda a la reconstrucción estructural de la piel aplicándolo en heridas de $1 \mathrm{~cm} 2$ infringidas a 48 ratones albinos, agrupados en cuatro tratamientos; Quitosano concentraciones de 0.15 y $0.30 \%$, producto cicatrizante (Ketanserina al $2 \%$ y blanco (testigos sin tratamiento). Los resultados permitieron afirmar que el gel de quitosano en ambas concentraciones puede ser utilizados en la 
regeneración de heridas, reduciendo en un 50\% el tiempo de cicatrización.

No obstante, en la revisión realizada se encontró un ensayo clínico fase II, aleatorizado y no enmascarado que incluyó 70 pacientes a cuyos integrantes se les lavó la herida con solución salina fisiológica y se les aplicó peloide durante 15 minutos; y de control (GC), tratados solamente con suero fisiológico yodado. En conclusión, el alto contenido de sulfuro de hidrógeno del peloide lo convierte en una sustancia bactericida y fungicida, por lo cual se utiliza como antiséptico moderado y antiparasitario; y en forma de lociones o aceites, contra el acné y la sarna, alcanzando el cierre de la herida en un 90\% de los pacientes tratados con este método. (Ramírez, R. R., Salazar, J. C., Tuero, J. H., Pineda, M. M., \& Quiala, J. G. 2004)

Castro,C.C.(2013), Ilevoacabounarevisiónde la literatura que tuvo como objetivo investigar sobre los efectos de las diferentes vitaminas (liposolubles e hidrosolubles) en el proceso de prevención y curación de las heridas de tipo agudo, concluyendo que las vitaminas son instrumentos terapéuticos en la prevención y tratamientos de las heridas y cada una tiene su rol en el proceso de la cicatrización. La suplementación multivitamínica mixta con otros nutrientes modula la cicatrización de las heridas.

Un estudio realizado por Amin MA, AbdelRaheem IT (2014), tuvo como objetivo desarrollar un apósito para heridas cargado de veneno de abeja con efectos curativos y antiinflamatorios mejorados para ser examinado en ratas diabéticas. Se desarrollaron diferentes preparaciones de alcohol polivinílico (PVA), apósito para heridas a base de matriz de hidrogel de quitosano (Chit) que contiene veneno de abeja (BV) utilizando el método de congelacióndescongelación. Se determinaron las propiedades mecánicas tales como fracción de gel, relación de hinchamiento, resistencia a la tracción, porcentaje de alargamiento y pH superficial. Se evaluaron las actividades farmacológicas, incluida la cicatrización de heridas y los efectos antiinflamatorios, además de la irritación cutánea primaria y las pruebas de penetración microbiana. Como resultado se obtuvo que farmacológicamente, el apósito para heridas cargado de veneno de abeja, mostró una curación acelerada de las heridas hechas en ratas diabéticas.

Todos los tratamientos revisados tienen fines específicos a través de los cuales puedan llevar a cabo con diversos tratamientos la curación de la herida. Cada uno de estos lo hacen a través de diversos mecanismos que le permiten aproximarse a la curación eficaz de las heridas impidiendo la progresión del daño en el tejido y la promoción de la integridad cutánea potenciando los mecanismo fisiológicos que le permiten al ser humano recuperar su estado de bienestar; es importante resaltar que cada uno de las terapias mencionadas anteriormente, tienen una profundización escasa en investigación, y por ello es necesario hacer énfasis en la exploración específica de los mecanismos de acción de cada terapia, y cómo funcionan en el ser humano, de tal forma que se potencien los tratamientos que hacen parte de la medicina tradicional, se implementen estas terapias de manera segura en la población y con sustento científico sólido, y se asegure la integración progresiva de este tipo de procedimientos a los sistemas de salud. 


\section{Conclusiones}

Esta revisión de literatura permitió identificar, la diversidad de tratamientos no convencionales utilizados en el manejo de las heridas como son: (miel, larvas, plantas medicinales, barro, entre otros) y evidenciar que cada día aumenta el número de estudios e investigaciones que buscan una sustentación científica para la utilización de éste tipo de tratamientos.

Los tratamientos no convencionales descritos presentan ventajas (producto de sus propiedades físicas y químicas) que se pueden resumir en: mayor acción en el desbridamiento, alta efectividad antiséptica y antimicrobiana, promoción del tejido de granulación y mantenimiento del ambiente húmedo en la herida.
Es indispensable que Enfermería esté actualizada en cuanto a las alternativas de tratamiento aquí descritas para curar las heridas ya que son las enfermeras y los enfermeroslosquedeunamanerauotratienen el primer vínculo con el paciente, le ayudan con una necesidad insatisfecha, trabajan en favor de la promoción y la prevención, son educadoras y educadores, líderes, brindan conocimientos con sustento científico y están atentas a prestar cuidados de calidad para la mejoría del estado de salud reconociendo en el sujeto prácticas de autocuidado y ayudando con su fortalecimiento permanente.

\section{Referencias Bibliográficas}

1. Abd Jalil MA, Kasmuri AR, Hadi H. (2017). Stingless Bee Honey, the Natural Wound Healer: A Review. Skin Pharmacol Physiol. 2017;30(2):66-75. doi: 10.1159/000458416. Epub 2017 Mar 15. PMID: 28291965.

2. Amin MA, Abdel-Raheem IT. (2014). Accelerated wound healing and anti-inflammatory effects of physically cross linked polyvinyl alcohol-chitosan hydrogel containing honey bee venom in diabetic rats. Arch Pharm Res. 2014 Aug;37(8):1016-31. doi: 10.1007/s12272-013-0308-y. Epub 2013 Nov 30. PMID: 24293065.

3. Andrade, A. P. (2012). El uso popular de Marañon (ANACARDIUM OCCIDENTALE L. -FRANZ EUGEN KÖHLER-1887). Amazonas.

4. Andrades, P., Sepúlveda, S., \& Gonzáles, J. (2004). Curación avanzada de heridas. revista chilena de cirguía, 396-403.

5. Arenas, D. J, D, (2020) Efecto cicatrizante del extracto etanólico de las hojas de Anonna muricata L. sobre heridas superficiales en Mus musculus var. albinus.

6. Ávila, C. M., \& Vázquez, R. T. (2006). larvoterapia: una antigua forma de curar heridas. BIOCIENCIAS, 7-14. 
7. Bang L, Bunting C, Molan P. (2003) The effect of dilution on the rate of hydrogen peroxide production in honey and its implications for wound healing. T. J. Alt. Compl. Med. Volumen 9, Número 2: P. 267-73

8. Bilsel, Y., Bugra, D., Yamaner, S., Bulut, T., Cevikbas, U., \& Turkoglu, U. (2002). Could Honey Have a Place in Colitis Therapy? Effects of Honey, Prednisolone, and Disulfiram on Inflammation, Nitric Oxide, and Free Radical Formation. Dig Surg, 306-312.

9. Bradshaw, C. E. (2011). Anin vitro comparison of the antimicrobial activity of honey, iodine and silver wound dressings. biosciencie Horizons, 61-70.

10. Buratti S, Benedetti S, Cosio. (2007) Evaluation of the antioxidant power of honey, propolis and royal jelly by amperometric flow injection analysis. Talanta. Volumen 71, Número $3: 1387-92$

11. Calderon, M. d., Figueroa, C. S., Arias, J. S., Sandoval, A. H., \& Torre, F. O. (2015). Terapia combinada con miel de Ulmo (Eucryphia cordifolia) y ácido ascórbico en úlceras venosas. Rev. Latino-Am. Enfermagem, 259-266.

12. Cook, M. P. (2020). Miel en el tratamiento de heridas:¿ Creencia o realidad?. Revista Horizonte de Enfermería, 19(1), 81-86.

13. Campos, D. C., Graveto, J. M., \& Silva, M. A. (2009). A aplicação do mel no tratamento. Revista Referencia, 117-124.

14. Castro, C. C. (2013). En la naturaleza esta la respuesta: "micronutrientes: las vitaminas agentes terapeuticos de las heridas". enfermeria global , 274-289.

15. Gentil GI, Smirova P (2009) Larvaterapia. Revisión sistemática de evidencia científica. Revista Internacional de Ciencias Podológicas. Volumen 3, nùmero 1: p. 45-52.

16. Girao, R. A. (2014). la miel en el tratamiento de heridas. Cantabria: Universidad de Cantabria.

17. Horobin, K.M.Shakesheff, S.Woodrow, C.Robinson, \& D.I.Pritchard. (2003). Maggots and wound healing: an investigation of the effects of secretions fromLucilia sericatalarvae upon interactions between human dermal fibroblasts and extracellular matrix components. British Journal of Dermatology, 923-933.

18. Ingle R, Levin J, Polinder K. (2006) Wound healing with honey - a randomised controlled trial. S Afr Med J. Volumen 96, Número 9: p. 831-835. [consultado 28 de abril de 2016] Disponible en: www.ajol.info/index.php/samj/article/download/13815/15782 
19. Inocente Camones, M. A. (2020). Actividad cicatrizante de seis extractos hidroalcohólicos de plantas en heridas incisas de Rattus norvegicus albinus.

20. Israili ZH. Antimicrobial properties of honey. Am J Ther. 2014 Jul-Aug;21(4):304-23. doi: 10.1097/MJT.Ob013e318293b09b. PMID: 23782759.

21. Jarczyk, G., Jackowski, M., Szpila, K., Boszek, G., \& Kapelaty, S. (2008). Use of Lucilia sericata blowfly maggots in the treatment of diabetic feet threatened with amputation. vía médica, 4255.

22. Jimenez, C. E. (2008). curación avanzada de heridas . revista colombiana de cirugía , 146-155.

23. Lavandera RI. (2011) Curación de heridas sépticas con miel de abejas . Revista Cubana de Cirugía Volumen 50, Nùmero 2: p. 187-196

24. Leininger, M. (1999). CUIDAR A LOS QUE SON DE CULTURAS DIFERENTES REQUIERE EL CONOCIMIENTO Y LAS APTITUDES DE LA ENFERMERÍA TRANSCULTURAL. cultura de los cuidados, 5-8.

25. Marshall C, Queen J, Manjooran J. (2005) Honey vspovidone iodine following toenail surgery. Wounds UK. Volumen 1, nùmero 1:p. 10-18 [consultado 28 mayo de 2016] Disponible en: http://www.wounds-uk.com/journal-articles/honey-vs-povidone-iodine-following-toenailsurgery-2

26. Martínez-P, S. (2020). Actualización sobre terapia larval en heridas. Enfermería investiga, 5(3), 5-7.

27. Ministerio de Agricultura y Desarrollo Rural Colombia. (2011). plantas aromáticas y medicinales, enfermedades de inportancia y su uso terapeútico. Bogotá D.C.: Produmedios.

28. Muñoz GF. (2006) Cuidados para la curación de heridas y quemaduras. España: Hospital Asepeyo; p.18-30.

29. Nicácio Da Silva, P., Alves de Almeida, O., \& Rocha, I. C. (2014). Terapia tópica en el tratamiento de las heridas crónicas. enfermería global, 33-45.

30. Okoli, C., Akah, P., \& Okoli2, A. (2007). Potentials of leaves of Aspilia africana (Compositae) in wound care: an experimental evaluation. Biomedcentral , 1-7.

31. Orellana Espinoza, M. I., \& Encalada Torres, R. S. (2019). Efectividad de apósitos Con miel de Abeja en el manejo de heridas crónicas en la mejora de la repitelización del tejido 
32. Oryan, A., Alemzadeh, E., \& Moshiri, A. (2015). Biological properties and therapeutic activities of honey in wound healing: A narrative review and meta-analysis. Journal of Tissue Viability, ELSEVIER, 1-21.

33. PIRIZ, M., LIMA, C., JARDIM, V., MESQUITA, M., SOUZA, A., \& HECK, R. (2014). Plantas medicinais no processo de cicatrização de feridas: uma revisão de literatura. Rev. Bras. Pl. Med, 628-636.

34. Ramirez, R. R., Salazar, J. C., Tuero, J. H., Pineda, M. M., \& Quiala, J. G. (2004). PELOIDOTERAPÍA EN LAS HERIDAS QUIRURGICAS INFECTADAS. Medisan, 32-38.

35. Richardson, M. (2004). The benefits of larval therapy in wound care. art\&sciencetissue viability supplement, 70-76.

36. Robson, V., Dodd, S., \& Thomas, S. (2009). Standardized antibacterial honey (Medihoney) with standard therapy in wound care: randomized clinical trial. JOURNAL OF ADVANCED NURSING, 565-575.

37. Rodríguez Diez, M. (2020). Terapia de desbridamiento larval. https://gredos.usal.es/ handle/10366/144102

38. Sánchez, H. F., Lozano, A. Y., Méndez-Gómez, E., Vázquez, A. E., Hernández, M. d., \& Lizárraga, A. E. (2014). Evaluación in vivo del efecto cicatrizante de un gel a base de quitosano obtenido de exoesqueleto de camarón blanco Litopenaeus vannamei. Rev. Colomb. Biotecnol.

39. Schencke, C., Salvo, J., Vasconcellos, A., \& Sol, M. d. (2013). Estudio Comparativo de la Cicatrización en Quemaduras con Tratamiento en Base a Miel de Ulmo (Eucryphia cordifolia) y Vitamina C oral versusHidrogel en Cobayos (Cavia porcellus). Int. J. Morphol, 839-844.

40. Sherman, R. A. (2013). Maggot Therapy for Treating Diabetic Foot Ulcers Unresponsive to Conventional Therapy. DIABETES CARE, 445-451.

41. Silva, R. S., Matos, L. S., Araújo, E. C., Paixão, G. P., Costa, L. E., \& Pereira., Á. (2014). Prácticas populares en salud: autocuidado con heridas de usuarios de plantas medicinales. revista enfermagem Uerj, 389-395.

42. Steenvoorde, P., Jacobi, C. e., Doorn, L. V., \& Oskam., J. (2007). Maggot debridement therapy of infected ulcers: patient and wound factors influencing outcome a study on 101 patients with 117 wounds. The Royal College of Surgeons of England, 596-602. 
43. Sulaeva, I., Henniges, U., Rosenau, T., \& Potthast, A. (2015). Bacterial cellulose as a material for wound treatment: Properties and modifications. A review. Biotechnology Advances, 1 547- 157

44. Téllez, G. A., Acero, M. A., Pineda, L. A., \& Castaño, J. C. (2012). Larvaterapia aplicada a heridas con poca carga de tejido necrótico y caracterización enzimática de la excreción, secreción y hemolinfa de larvas. biomédica, 312-320.

45. Tonks AJ, Cooper RA, Jones KP, Blair S, Parton J, Tonks A. (2003) Honey stimulates inflammatory cytokine production from monocytes. Cytokine. 21(5):242-7 [consultado 23 de abril de 2016] Disponible en: https://www.ncbi.nlm.nih.gov/pubmed/12824009

46. Waymant, J., Nirojogit, V., Walker, A., Sowinskia, A., \& Walkera, M. A. (2000).

The cost effectiveness of larval therapy in venous ulcers. Journal of Tissue Viability, 91-94.

47. Zhang, Q., Fong, C. C., Yu, W. K., Chen, Y., Wei, F., Koon, C. M., .. Yang., M. (2012). Herbal formula Astragali Radix and Rehmanniae Radix exerted wound healing effect on human skin fibroblast cell line Hs27 via the activation of transformation growth factor (TGF) pathway and promoting extracellular matrix (ECM) deposition. Phytomedicine, ELSEVIER, 9-16. 